\title{
Two-Photon Direct Laser Writing Beyond the Diffraction Limit Using the Nanopositioning and Nanomeasuring Machine
}

\author{
Laura Mohr-Weidenfeller ${ }^{1}\left[\right.$ [ Annika-Verena Häcker ${ }^{2} \cdot$ Carsten Reinhardt $^{3} \cdot$ Eberhard Manske $^{2}$
}

Received: 20 December 2020 / Revised: 17 February 2021 / Accepted: 24 February 2021 / Published online: 5 April 2021

(c) The Author(s) 2021

\begin{abstract}
Since the first realization of two-photon direct laser writing (DLW) in Maruo et al. (Opt Lett 22:132-134, 1997), the manufacturing using direct laser writing techniques spread out in many laboratories all over the world. Photosensitive materials with different material properties open a new field for micro- and nanofabrication. The achievable structuring resolution using this technique is reported to be sub-100 nm (Paz et al. in J. Laser Appl. 24:042004, 2012), while a smallest linewidth of $25 \mathrm{~nm}$ could be shown in Tan et al. (Appl Phys Lett 90:071106, 2007). In our approach, the combination of DLW with the nanopositioning and nanomeasuring machine NMM-1 offers an improvement of the technique from the engineering side regarding the ultra-precise positioning (Weidenfeller et al. in Adv Fabr Technol Micro/Nano Opt Photon XI 10544:105440E, 2018). One big benefit besides the high positioning resolution of $0.1 \mathrm{~nm}$ is offered by the positioning range of $25 \mathrm{~mm} \times 25 \mathrm{~mm} \times 5 \mathrm{~mm}$ (Jäger et al. in Technisches Messen 67:319-323, 2000; Manske et al. in Meas Sci Technol 18:520-527, 2007). Thus, a trans-scale fabrication without any stitching or combination of different positioning systems is necessary. The immense synergy between the highly precise positioning and the DLW is demonstrated by the realization of resist lines and trenches whose center-to-center distance undergoes the modified diffraction limit for two-photon processes. The precise positioning accuracy enables a defined distance between illuminated lines. Hence, with a comparable huge width of the trenches of $1.655 \mu \mathrm{m}$ due to a low effective numerical aperture of 0.16 , a resist line of $30 \mathrm{~nm}$ between two written trenches could be achieved. Although the interrelationships for achieving such narrow trenches have not yet been clarified, much smaller resist lines and trench widths are possible with this approach in the near future.
\end{abstract}

Keywords Direct laser writing $\cdot$ Nanopositioning and nanomeasuring machine $\cdot$ Two-photon absorption $\cdot$ Laser power controlling

\section{Introduction}

In the last decades, two-photon absorption (2PA) has become a very promising tool for three-dimensional micro- and nanofabrication [2]. In this fabrication technique, a tightly focused ultrashort pulsed laser beam is used to induce energetic transitions in a photosensitive material [7]. Due to the

Laura Mohr-Weidenfeller

laura.weidenfeller@tu-ilmenau.de

1 Microsystems Technology Group, Ilmenau University of Technology, Max-Planck-Ring 12, Ilmenau, Germany

2 Institute for Process Measurement and Sensor Technology, Ilmenau University of Technology, Gustav-Kirchhoff-Str. 1, Ilmenau, Germany

3 Bremen University of Applied Science, Neustadtswall 30, Bremen, Germany high spatial and temporal focusing, the needed energy $\Delta E$ for the transition can be provided by the simultaneous absorption of two photons with the energy $\frac{\Delta E}{2}$ instead of one photon with the energy $\Delta E$. In contrast to one-photon absorption, which follows the Beer-Lambert law, the 2PA processes only takes place in the focal region [7]. Hence, by a relative movement between the focal spot and the photosensitive material, a three-dimensional structure can be created $[2,4]$. Several artistical, medical, and optical applications were demonstrated in the last years [8-10]. The lithographic resolution is given with around $100 \mathrm{~nm}$ for line with constant width [11], even though certain suspended lines with thinnest parts down to $25 \mathrm{~nm}$ could be achieved [3]. Super-resolution techniques like stimulated emission depletion STEDDLW or SLM-assisted DLW (SLM, spatial light modulator) decrease the dimension of constantly written lines to $55 \mathrm{~nm}$ [12]. Due to the behavior of light, some groups predict the 
validation of a modified diffraction limit, even though the writing is a serial process [13]. The reason is motivated in a threshold behavior in combination with a memory of the photoresist, which sums up the induced illumination doses below the threshold [13]. In consequence, the fabrication of structures and trenches between these structures could only be realized by super-resolution DLW techniques [13].

Besides the lithographic resolution, the positioning resolution and the repeatability are limiting factors in processing precise structures [4]. The positioning resolution depends on the scanning mode and therewith on the applied positioning systems [14]. The most precise positioning systems that are used for STED-DLW offer repeatabilities of around $10 \mathrm{~nm}$ [15]. Nevertheless, if extended areas should be addressed with the positioning systems, the drawbacks in precision must be taken into account. One of the large area fabrication systems addresses a positioning volume of $150 \mathrm{~mm} \times 150 \mathrm{~mm} \times 4 \mathrm{~mm}$ with a resolution of $0.5 \mathrm{~nm}$ per direction, but the repeatability is given with $75 \mathrm{~nm}$ for the linear stages in $x$ - and $y$-direction and $200 \mathrm{~nm}$ in $z$-direction [14]. Accordingly, the precision of the positioning systems is in the same range or even worse than the lithographic resolution of the DLW technique. To improve the precision of two-photon processes by means of positioning, nanopositioning and nanomeasuring machines are very promising.

\section{Experimental Setup}

The experimental setup for two-photon DLW with the use of the nanopositioning and nanomeasuring machine NMM-1 is depicted in Fig. 1. The main part for the 2PA-processes in the photosensitive material is a Ti:sapphire oscillator (Spectra Physics) with a central wavelength of $801 \mathrm{~nm}$, a repetition rate of $85 \mathrm{MHz}$, and a pulse duration of approximately $43 \mathrm{fs}$. The beam passes a half waveplate and a polarizing beam splitter cube, which enables adjusting the polarization

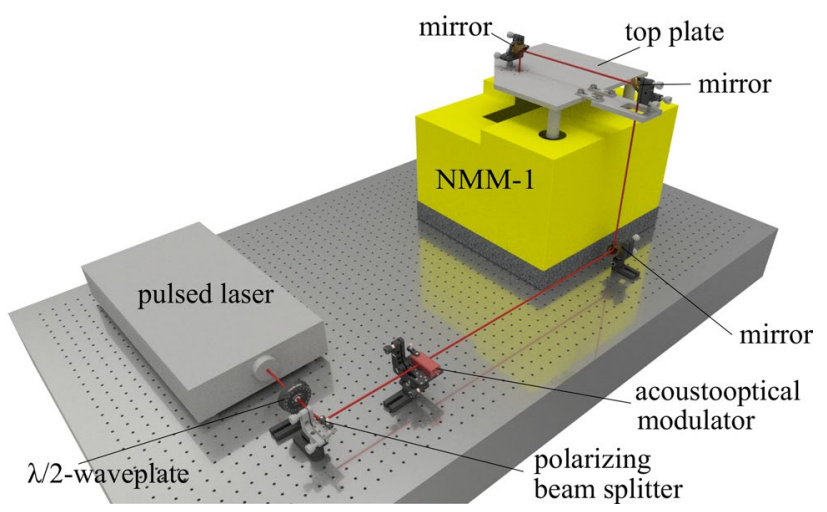

Fig. 1 Setup for two-photon DLW with the nanopositioning and nanomeasuring machine on the one hand and attenuating the power of the laser beam on the other hand. Subsequently, an acousto-optical modulator (Pegasus Optik $\mathrm{GmbH}$ ) is used to shutter the laser beam by guiding the first diffraction order of the beam to mirrors. Finally, the beam enters the objective and is focused onto the sample stage of the NMM-1.

The distance between the focal spot and the sample with the photosensitive material is measured and controlled by a laser focus sensor that was developed for the use with nanopositioning and nanomeasuring machines [16]. This laser focus sensor works contact free with a wavelength of $650 \mathrm{~nm}$ to avoid an influence on the photosensitive material, which is typically designed for shorter wavelengths in the range of $400 \mathrm{~nm}$ or less. Due to the sensor's optical properties, the numerical aperture for the used objective is determined, which leads to a reduced $N A$ for the used femtosecond laser beam of only 0.16 . The sensor's measuring velocity goes up to $6 \mathrm{~mm} / \mathrm{s}$ with a reproducibility of $2 \mathrm{~nm}$ [16]. Such a high precision is achieved by a consequent compliance of the Abbe Comparator Principle, which is not only implemented for the laser focus sensor but also for the movement of the sample stage.

\subsection{Principle of Nanopositioning}

For the precise positioning, a resolution of $0.1 \mathrm{~nm}$ and a repeatability of less than $1 \mathrm{~nm}$ [17], over a large measuring volume of $25 \mathrm{~mm} \times 25 \mathrm{~mm} \times 5 \mathrm{~mm} \mathrm{[5,6]} \mathrm{are} \mathrm{the} \mathrm{center}$ pieces of the hole setup. The basic measurement approach is, like mentioned above, the Abbe Comparator Principle, which says that the measuring apparatus is to be arranged in such a way that the distance to be measured is a straight-line extension of the graduation used as a scale [18]. To realize this highest precision, it is necessary to apply the Abbe Comparator Principle in all three coordinate axes. The Abbe error in three dimensions can be described as

$\Delta l_{i}=l_{\mathrm{off}, i} \cdot \sin \left(\alpha_{i}\right) \quad i \in x, y, z$

and is minimized in the measuring setup.

Mostly for nanopositioning, piezoelectrical devices are used. Even if the positioning with these piezoelectrical devices is comparably precise, they can just address a small range. In order to achieve larger positioning ranges, so-called stitching methods must be used. A different actuation was chosen for the NMM-1. For the realization of the positioning, voice coils in combination with anti-friction bearings are integrated [19].

The NMM-1 combines a complex interferometric length and angle measurement, with a three-dimensional platform positioning. For the supply and measurement acquisition, as well as the measurement evaluation of the various components, four different units are integrated: the laser unit with 
frequency stabilized laser, the interferometer unit for the acquisition and evaluation of the input signals from the interferometers, the digital signal processor (DSP) for all processed laser signals as well as the environmental signals and the calculated position signals, and the motor unit to generate the input signals for the voice coils.

The central module of the measured value acquisition and processing is the DSP. It is responsible for the measuring sequence control, the position and angle control, as well as for the manipulated variables control. For the positioning of the sample table, the enormous amounts of measurement and positioning data must be recorded and processed in real time. For this purpose, the measurement data acquisition is at $6.25 \mathrm{kHz}$. The movement of the sample stage occurs in a closed-loop controlled manner [16]. In order to achieve a high resolution despite the real-time capability of the system by using high-precision fiber coupled planar interferometers for the measurement system [20,21], a combination with 13-bit $\mathrm{AD}$ converters has been used [22].

\section{Theory of Diffraction Limit for 2PA}

Regarding a limit for the resolution of resist lines and trenches fabricated by means of DLW, Fischer et al. [13] predict a modified Abbe Criterion as diffraction limit for 2PA. As for 2PA, the squared Gaussian intensity profile has to be taken into account, the Abbe criterion is extended by the narrowing factor $\sqrt{2}$ compared to the regular Gaussian intensity. A DLW process for two or more lines is hardly comparable with the diffraction produced by a grid, but more like a two adjacent objects, the Rayleigh criterion seems to be more convenient to describe the process. The modified 2PA-Rayleigh diffraction limit is then given by

$d=0.61 \cdot \frac{\lambda}{\sqrt{2} \cdot N A}$

whereas $d$ is the minimum distance between the two objects, which means in this case the center-to-center distance between the illuminated lines, $\lambda$ is the wavelength and $N A$ is the numerical aperture. Their motivation is a threshold behavior of photoresist during the illumination process so that a certain amount of illumination dose is needed to start a reaction [23]. This threshold behavior defines the line width for a given illumination time, laser power, and focal spot size (see Fig. 2). When the dose is below this threshold, the amount of energy is stored in the photoresist and subsequent illumination processes add on this amount of energy. Fischer et al. [13] call this effect the resist memory. For the given setup, the 2PA Rayleigh diffraction limit is determined by $d=2.16 \mu \mathrm{m}$.

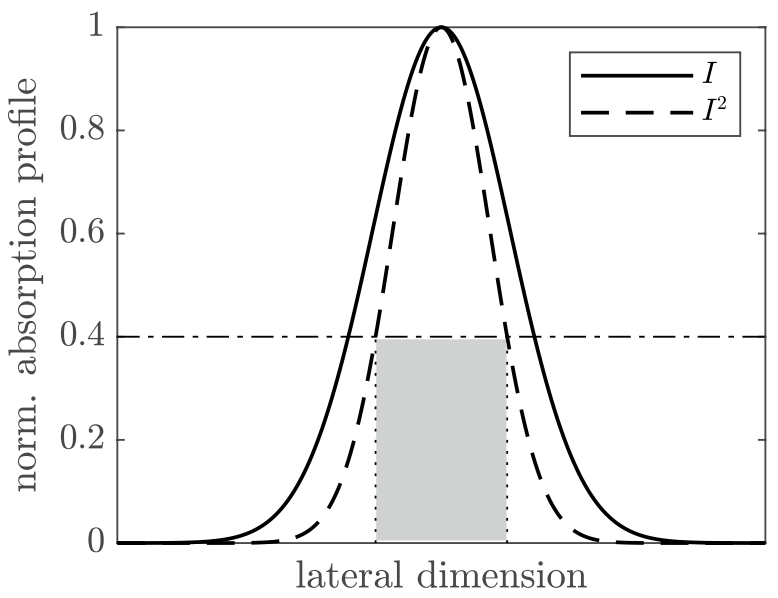

Fig. 2 Normalized absorption profile for one-photon absorption (solid line) and the squared intensity profile for two-photon absorption (dashed line) for a Gaussian beam with an arbitrary focal spot size in lateral dimension. The threshold for a reaction in the photoresist is exemplarily given by 0.4 of the total amount of laser power (dash-dotted line), which leads to a line width indicated by the dotted lines and the gray rectangle

\section{Fabrication of Small Resist Lines}

To prove the 2PA diffraction limit, samples were prepared as followed: a $130 \mu \mathrm{m}$ thin borosilicate glass was treated with hexamethyldisilazane for adhesion promotion and then spin-coated with AZ1505 (MicroChemicals $\mathrm{GmbH}$, Ulm, Germany). A prebake for $1 \mathrm{~min}$ at $115^{\circ} \mathrm{C}$ was applied. In first experiments, the center-to-center distance between illuminated lines was systematically reduced from $10 \mu \mathrm{m}$ to $1 \mu \mathrm{m}$ in steps of $1 \mu \mathrm{m}$. The diameter of the femtosecond laser spot at the sample surface was measured to be $4.6 \mu \mathrm{m}$. The illumination dose was chosen in the way that the achieved trenches in the positive resist after development have a width of $1.655 \mu \mathrm{m}$ to assure less line edge roughness. Assuming that the diffraction limit is valid for the process, the trenches that occur in positive resist AZ1505 at the illuminated lines should be separated by resist lines for bigger distances between the illuminated regions. For trenches resulting from illuminated lines with a distance of $2 \mu \mathrm{m}$ and less, the diffraction limit predicts that no separation could be observed and the trenches should overlap resulting in one big trench in the resist without separation of a resist line.

In Fig. 3, the achieved trenches and resist lines are shown. The combination of two-photon DLW with the NMM-1 offers best conditions for a precise positioning and therefore for further very exact investigation regarding the smallest possible distances between the illuminated lines. The nominal distance between the illuminated lines, which means the distance between the coordinates of the trajectories for the lines, was decreased successively. 


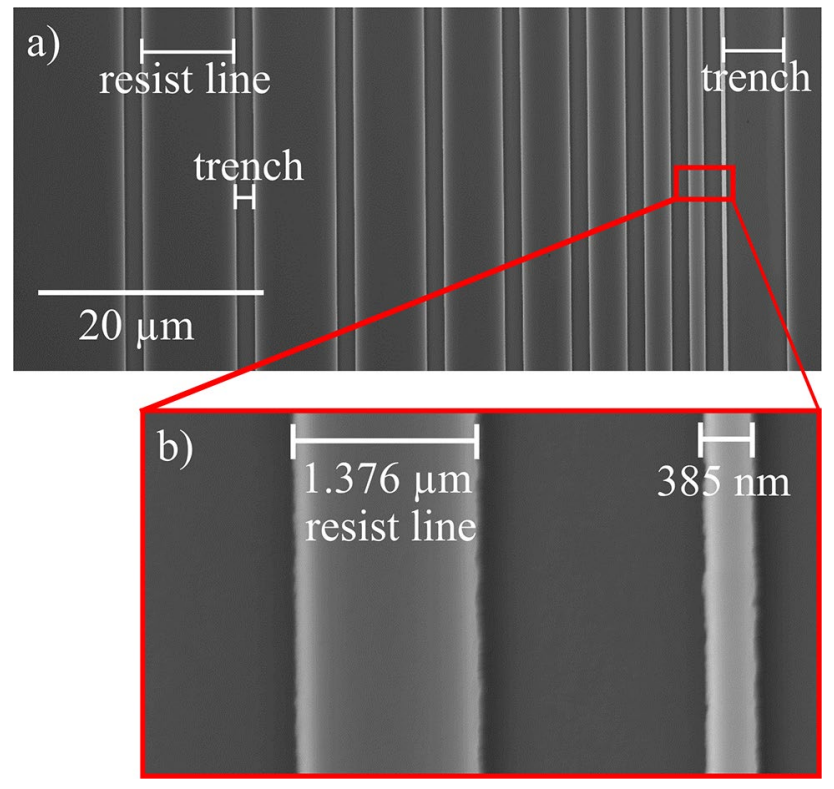

Fig. 3 a Trenches written in AZ1505 with a nominal distance between illuminated lines that was systematically reduced from 10 $\mu \mathrm{m}$ to $1 \mu \mathrm{m}$ in steps of $1 \mu \mathrm{m}$. The resist lines between the trenches decrease down to $385 \mathrm{~nm}$ for a center-to-center distance of $2 \mu \mathrm{m}$. For a nominal distance between the illuminated lines of $2 \mu \mathrm{m}$, just one broad trench is visible. b Detailed view of the resist lines for nominal distances of $3 \mu \mathrm{m}$ and $2 \mu \mathrm{m}$ with resulting resist lines of $1.376 \mu \mathrm{m}$ and $385 \mathrm{~nm}$

In the used setup, the very precise positioning of the NMM-1 allows to reduce the center-to-center distance in much finer steps. For further investigations on possible limits regarding the distance between illuminated lines, a step size of $5 \mathrm{~nm}$ was chosen. The results can be seen in Fig. 4 that shows resist lines between the trenches that are further decreased. The tiniest resist line that could be achieved without any discontinuities was realized with a width of $38 \mathrm{~nm}$ for a nominal distance of $1.695 \mu \mathrm{m}$. Even a 30-nm resist line was found, but it exhibits some discontinuities.

\section{Discussion}

Obviously, the experimental results show that achieved trenches and resist lines and thereby the associated nominal distances are below the predicted diffraction limit for two-photon processes. If the resist has a memory for illuminations below the threshold, the absorption profiles corresponding to Fig. 2 would sum up according to the superposition principle for the illumination of two adjacent lines. In Fig. 5, the normalized two-photon absorption profiles for the DLW process with maintained Rayleigh distance are shown. The normalization of the profiles facilitates the comparison to the theory and focuses on the essential information. The

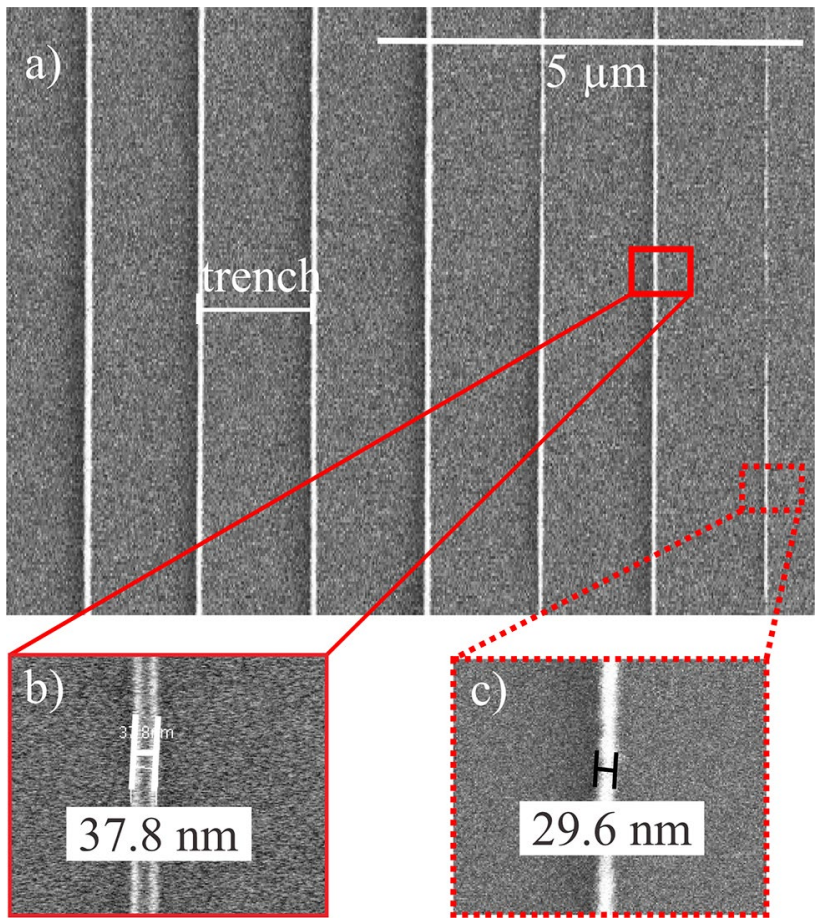

Fig. 4 a Written trenches with nominal distances from $1.72 \mu \mathrm{m}$ to $1.69 \mu \mathrm{m}$ decreasing $5 \mathrm{~nm}$ per illuminated line. The resulting resist lines are shown in detail in $\mathbf{b}$ with a $38-\mathrm{nm}$ resist line for a centerto-center distance of $1.695 \mu \mathrm{m}$ and $\mathbf{c}$ with a $30-\mathrm{nm}$ resist line for a center-to-center distance of $1.690 \mu \mathrm{m}$

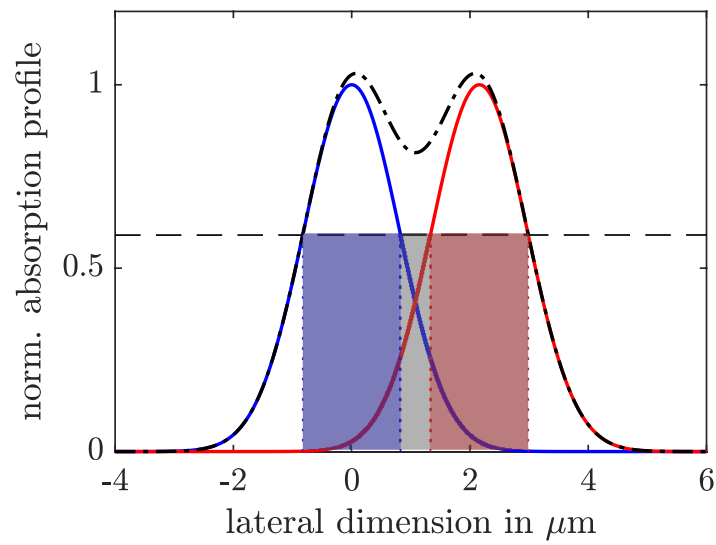

Fig. 5 Normalized absorption profiles for two illuminated lines for two-photon DLW (blue and red line) that are written with the theoretical Rayleigh distance of $2.16 \mu \mathrm{m}$. The width of $1.655 \mu \mathrm{m}$ for each of the resulting trenches is indicated by the blue and red dotted lines and rectangles. The threshold (dotted line) is determined by the measured width of the experimentally achieved trenches of $1.655 \mu \mathrm{m}$. The superposition of both absorption profiles is shown by the dash-dotted line and the theoretically achieved trench is indicated by the overlaying gray rectangle 
superposed absorption profile resulting from both individual illuminations is calculated and the absorption threshold for the process is derived from the achieved width of the illuminated trenches. In this case, the superposition absorption profile exhibits a dip, but in the range between the illuminated regions it is completely above the reaction threshold. In consequence, following the theory of resist memory, this would illuminate the resist in the blue, red, and gray area with enough energy for the absorption process remaining in one broad trench in the resist.

For a distance of around $2.55 \mu \mathrm{m}$ between the center of the illuminated lines, the simulations show separated resulting trenches due to a superposition profile that falls below the threshold in a small region (see Fig. 6). The simulation also shows that in this case the width of the resulting trenches (indicated by the red and blue rectangle) in the resist would be broadened (indicated by the gray rectangles) compared to lines that are written with a bigger distance to each other. Such a broadening in the linewidth was not obtained in the experimental part of this work (see Figs. 3 and 4). At this point, it is obvious that the theory of the remembering resist should be reviewed. In Fig. 7, the normalized two-photon absorption profiles for the experimentally shown illumination with a resulting resist line of $38 \mathrm{~nm}$ (according to Fig. 4) are shown. As in Fig. 5, again the whole range from the left edge of the blue indicated illumination line up to the right edge of the red indicated illumination line is induced by energy above the threshold if the superposition is assumed. However, as shown experimentally, even though the illuminated lines are quite close to each other, two well-defined trenches with a separation resist line with a width of $38 \mathrm{~nm}$ appear after development of the sample. The distance at

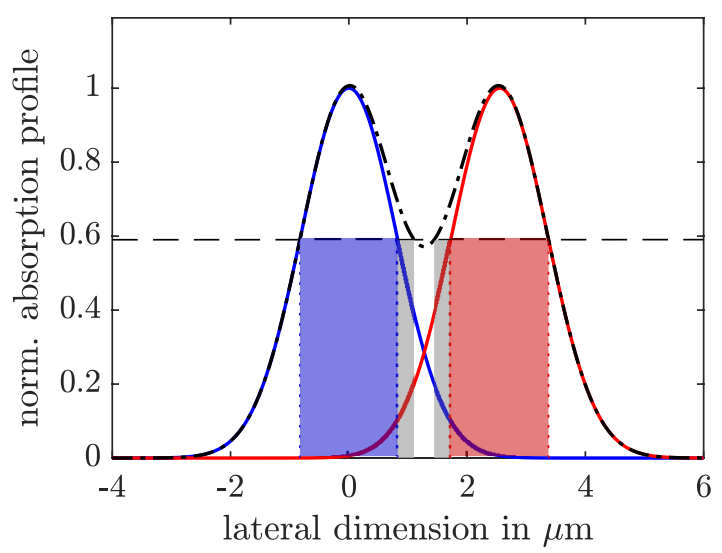

Fig. 6 Normalized absorption profiles for two illuminated lines for two-photon DLW (blue and red line) that are written with a distance of $2.16 \mu \mathrm{m}$. The dotted lines and the red and blue rectangles show the width of the resulting trenches of $1.655 \mu \mathrm{m}$. The gray rectangles indicate the broadening of the lines by assuming an illumination according to the superposition (dash-dotted line) and the threshold (dotted line)

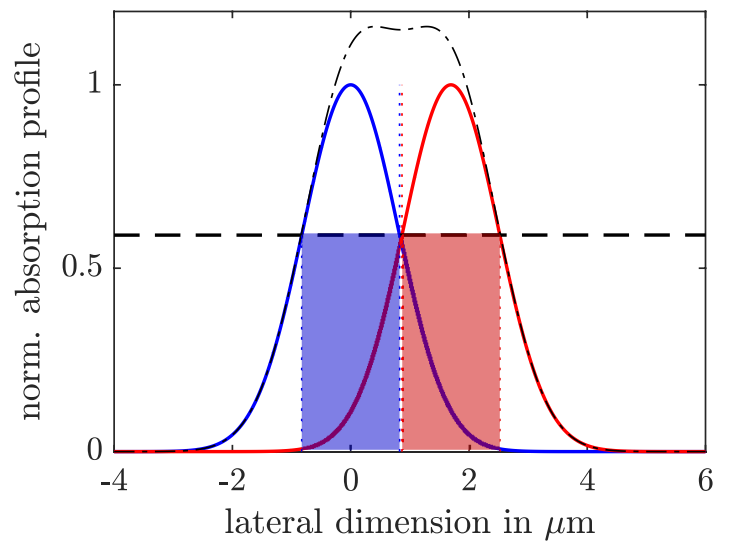

Fig. 7 Normalized absorption profiles for two illuminated lines for two-photon DLW (blue and red line) that are written with a distance of $1.695 \mu \mathrm{m}$ as shown in $4 \mathrm{~b}$. The dotted lines and the red and blue rectangles show the width of the resulting trenches of $1.655 \mu \mathrm{m}$. The superposition of both profiles is shown by the dash-dotted line and the threshold by the dotted line. The distance between the red and the blue dotted line in the simulation is $41 \mu \mathrm{m}$

threshold level between the indicated trenches in the simulation is $41 \mathrm{~nm}$, which is considerably conforming with the experimental results. With these results, we could show that a conventional 2PA-DLW process is able to create trenches and resist lines beyond the diffraction limit. Up to now, the diffraction limit was only broken by STED-DLW [13, 24].

\section{Conclusions and Outlook}

In conclusion, it was experimentally shown that by means of two-photon laser direct writing structures beyond the diffraction limit were realized. With the current setup, we should have a diffraction limit of $2.16 \mu \mathrm{m}$. Nevertheless, it was possible to write two adjacent illuminated lines with a distance of $1.695 \mu \mathrm{m}$, resulting in resist lines between the written trenches down to $30 \mathrm{~nm}$. This also leads to the result that the theory of the remembering resist should be reviewed. Theoretically, the smallest possible centerto-center distance should be given by the least dip in the superposition absorption profile according to Sparrow criteria [13], which should be investigated in future experiments. In this work, the illuminated regions and therefore the width of the resulting trenches in the positive resist, are comparably big caused by the low effective numerical aperture. A setup with an integrated beam expander promises line widths in the regime of the state of the art. As it can be seen in Fig. 4, discontinuities in the resist lines arised for smaller distances between the illuminated lines. To prevent the influences of fluctuating laser power on the width of the trenches and therefore the discontinuities, a new controlling concept should be investigated for 


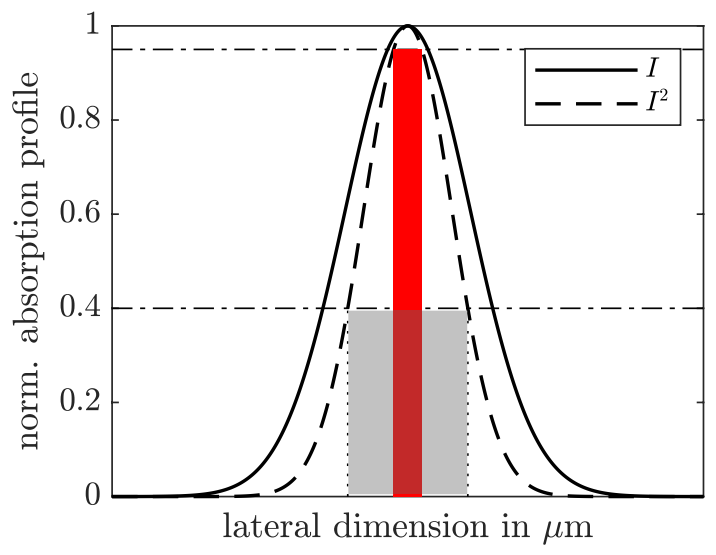

Fig. 8 Normalized absorption profiles by adjusting the threshold (dash-dotted lines) to a higher percentage, the resulting linewidth (gray rectangle and red rectangle, respectively) for the two-photon absorption profile (dashed line) can be reduced

the setup. Additionally, with a controlled laser power, the threshold for the illumination processes (see Fig. 2) can be adjusted finer, so that very small trenches would be possible by only using the peak of the absorption profile.

To push the linewidth to its minimum, the absorption profile has to be shaped by adjustments of the laser power and illumination time in order that the peak is slightly over the threshold. Shaping the absorption profile can be theoretically equalized with adjusting the threshold height. Therewith the lateral dimension of the reactive area on the resist is thinner (see Fig. 8). However, going closer to the threshold has the drawback that the writing process gets more sensitive to laser fluctuations [13]. Further, in the given setup, there are relative uncertainties of the laser power of around $\pm 7 \%$ in dependence of the applied voltage at the acousto-optical modulator. Thus, for writing at the threshold, some trenches would occurs due to enough illumination dose, others are illuminated below the threshold. To counteract this, the development of a control concept for the laser power is the next necessary step. At last, the design of more complex structures with DLW should be enabled by a controlled laser power, depending on the writing velocity.

Acknowledgements The authors gratefully acknowledge the support by the Deutsche Forschungsgemeinschaft (DFG) in the framework of Research Training Group Tip-and laser-based 3D-Nanofabrication in extended macroscopic working areas (GRK 2182) at the Technische Universität Ilmenau, Germany. C. Reinhardt acknowledges financial support from the DFG from the grant RE3012/4-1.

Funding Open Access funding enabled and organized by Projekt DEAL.

\section{Declarations}

Conflict of interest The authors declare that they have no conflicts of interest.

Open Access This article is licensed under a Creative Commons Attribution 4.0 International License, which permits use, sharing, adaptation, distribution and reproduction in any medium or format, as long as you give appropriate credit to the original author(s) and the source, provide a link to the Creative Commons licence, and indicate if changes were made. The images or other third party material in this article are included in the article's Creative Commons licence, unless indicated otherwise in a credit line to the material. If material is not included in the article's Creative Commons licence and your intended use is not permitted by statutory regulation or exceeds the permitted use, you will need to obtain permission directly from the copyright holder. To view a copy of this licence, visit http://creativecommons.org/licenses/by/4.0/.

\section{References}

1. Maruo S, Nakamura O, Kawata S (1997) Three-dimensional microfabrication with two-photon-absorbed photopolymerization. Opt Lett 22:132-134

2. Paz VF et al (2012) Development of functional sub-100 nm structures with 3D two-photon polymerization technique and optical methods for characterization. J Laser Appl 24:042004

3. Tan D et al (2007) Reduction in feature size of two-photon polymerization using SCR500. Appl Phys Lett 90:071106

4. Weidenfeller L et al (2018) Development of laser positioning system of high accuracy in the nanometer range. Adv Fabric Technol Micro/Nano Opt Photon XI 10544:105440E

5. Jäger G et al (2000) Nanomessmaschine zur abbefehlerfreien Koordinatenmessung (Nano Measuring Machine for Zero Abbe Offset Coordinate-measuring). TN-Technisches Messen 67:319-323

6. Manske E et al (2007) New applications of the nanopositioning and nanomeasuring machine by using advanced tactile and non-tactile probes. Meas Sci Technol 18:520-527

7. Sun H-B, Matsuo S, Misawa H (1999) Three-dimensional photonic crystal structures achieved with two-photon-absorption photopolymerization of resin. Appl Phys Lett 74:786-788

8. Yetisen AK et al (2016) Art on the Nanoscale and Beyond. Adv Mater 28:1724-1742

9. Chun-Yu Lin et al (2012) Investigation of two-photon excited fluorescence increment via crosslinked bovine serum albumin. Opt Express 20:13669-13676

10. Mangirdas Malinauskas et al (2010) Femtosecond laser polymerization of hybrid/integrated micro-optical elements and their characterization. J Opt 12:124010

11. Lei Zheng et al (2019) Nanofabrication of high-resolution periodic structures with a gap size below $100 \mathrm{~nm}$ by two-photon polymerization. Nanoscale Res Lett 14:1-9

12. Wollhofen R et al (2013) $120 \mathrm{~nm}$ resolution and $55 \mathrm{~nm}$ structure size in STED-lithography. Opt Express 21:10831-10840

13. Fischer J, Wegener M (2011) Three-dimensional direct laser writing inspired by stimulated-emission-depletion microscopy. Opt Mater Express 1:614-624

14. Weidenfeller L et al (2019) Laser-microfabrication with accurate positioning and metrological traceability. Adv Fabric Technol Micro/Nano Opt Photon XII 10930:109300L

15. Weidenfeller L (2020) Kombination von zweiphotonenbasiertem direktem Laserschreiben mit großflächiger und hochpräziser 
Nanopositionierung. TU Ilmenau Universitätsbibliothek, Ilmenau

16. Manske E, Jäger G, Hausotte T (2012) A multi-sensor approach for complex and large-area applications in micro and nanometrology. NCSLI Meas 7:44-50

17. Manske E et al (2012) Recent developments and challenges of nanopositioning and nanomeasuring technology. Meas Sci Technol 23:074001

18. Abbe E (1890) Meßapparate für Physiker. Z Instrum 10:446-448

19. Hausotte T (2010) Nanopositionier- und NanomessmaschinenGeräte für hochpräzise makro- bis nanoskalige Oberflächenund Koordinatenmessungen. TU Ilmenau Universitätsbibliothek, Ilmenau

20. Büchner $H$ et al (1992) Interferometrisches Meßverfahren zur berührungslosen und quasi punktförmigen Antastung von
Meßoberflächen/interferometric measuring technique for the contactless and quasi-pointlikescanning of measuring surfaces. TM-Technisches Messen 59(2):43-47

21. Jäger G (1999) Lasernanomesstechnik-Möglichkeiten, Grenzen und Anwendungen in der modernen Gerätetechnik. In: 44th International scientific colloquium. TU Ilmenau, 20 (23.09)

22. Manske E, et al. (2005) Nanopositioning and nanomeasuring machine for high accuracy measuring procedures of small features in large areas. In: Optical fabrication, testing, and metrology II, vol 5965. International Society for Optics and Photonics, p 596509

23. Fischer J (2012) Three-dimensional optical lithography beyond the diffraction limit. Karlsruher Institut für Technologie, Karlsruhe

24. Gan Z et al (2013) Three-dimensional deep sub-diffraction optical beam lithography with $9 \mathrm{~nm}$ feature size. Nat Commun 4:1-7 